\title{
Providing Evidence: Step by Step—a Letter from Associate Editor, Tobias Else, MD
}

\author{
Tobias Else ${ }^{1}$ \\ Published online: 7 December 2018 \\ (C) Springer Science+Business Media, LLC, part of Springer Nature 2018
}

Most endocrine tumors represent rare diseases and this is definitely true for pheochromocytoma, paraganglioma, and adrenocortical carcinoma. Even for the few endocrine tumors that are not rare, specific subtypes (e.g., anaplastic thyroid cancer) or the predisposing syndromes (e.g., multiple endocrine neoplasia type 1, von Hippel-Lindau disease) are certainly rare. The rarity of these diseases inherently challenges our ability to do impactful research, publish findings with broad application, and develop guidelines to provide evidence-based clinical care.

The challenges in care for patients with rare endocrine tumors are far too familiar to specialized physicians. There is a lack of evidence, and the approach to care is too often driven by personal experience and research from small case series. Even guidelines are often based on expert opinion and expert experience rather than on large-scale epidemiological studies. Therefore, most guideline recommendations will be supported by low to very low strength of evidence. For example, a moderate quality of evidence preferably requires randomized controlled trials (RCTs). Obviously, sufficiently powered RCTs are as rare as these diseases themselves. In the past, low or very low evidence recommendations have commonly become textbook knowledge, which then becomes difficult to overcome and correct. In practice, the absence of strong evidence leads to questionable guidelines and significant variation in care for patients with rare endocrine tumors.

The challenges in generating sufficient evidence are as onerous as the challenges in care, but there are some emerging solutions. One such obvious solution is to utilize larger consortia, such as the European Network for the Study of Adrenal Tumors (ENSAT) or the recently inaugurated AmericanAustralian-Asian Adrenal Alliance (A5), in order to increase

Tobias Else

telse@med.umich.edu

1 Division of Metabolism, Endocrinology and Diabetes, University of Michigan, Ann Arbor, MI, USA patient numbers in research and thereby enhance the strength of available evidence. These efforts take time and will slowly address some of the pertinent questions but only as a result of well-planned RCTs. Until then, there remains a need for the dissemination of local or national experiences from referral center retrospective and observational trials [1,2], particularly when exploring very detailed aspects of pharmacotherapy of rare endocrine cancers [3]. The current issue of Hormones and Cancer highlights an example of such an important national experience by featuring the Chilean Registry for Neuroendocrine Tumors [4]. Equally important sources of evidence are translational studies exploring the pathogenesis of endocrine tumors or those focusing specifically on patients with hereditary predisposition to certain tumors. Experiences from these ultra-rare populations have historically informed our understanding of disease, such as the approach to sporadic neuroendocrine tumors (e.g., MEN1), and shed light into the general pathogenesis of certain tumors (e.g., VHL and renal cell cancer). In the space of rare diseases, no evidence should be dismissed; this may even include exceptional case reports that are hypothesis generating or provide a potential basis of new diagnoses or therapies. Thus, Hormones and Cancer will now consider case reports that can significantly add to our understanding of disease processes and patient care relevant to rare neuroendocrine tumors.

Last but not least, there are challenges in publication of results pertinent to rare diseases. In the field of endocrine and oncological research, there is certainly a bias toward more prevalent diseases, such as diabetes or common malignancies. Additionally, the choice of journal has become more difficult with traditional flagship journals releasing additional subspecialty journals. The landscape of journals has also become more blurred by a rising number of obscure journals trying to (aggressively) garner manuscripts. Traditionally, researchers encounter difficulties publishing manuscripts with confirmatory, contradictory, or negative results. However, confirmatory and contradictory studies are important in order to increase - or question - the low level of evidence that is 
currently available for almost all aspects of rare diseases. Moreover, negative results can be equally informative, particularly, when related to the testing of an obvious hypothesis or evaluating outcomes of common practices.

Hormones and Cancer understands itself as a partner in solving these issues. The journal's scope includes all clinical, translational, and basic science aspects of hormone-producing and hormone-responsive tumors. For the field of endocrinology, it is fair to state that this definition not only includes malignant but also benign tumors and hormone excess syndromes. Most researchers and clinicians share an interest in all of these entities. The Editors and the Editorial Board are very familiar with the challenges of research of endocrine tumors and other rare diseases. Therefore, we welcome all manuscripts that increase the knowledge about endocrine tumors, particularly those that provide the step-by-step evidence that is needed to ultimately impact patient care.

Publisher's Note Springer Nature remains neutral with regard to jurisdictional claims in published maps and institutional affiliations.

\section{References}

1. Asai S, Katabami T, Tsuiki M, Tanaka Y, Naruse M (2017) Controlling tumor progression with cyclophosphamide, vincristine, and dacarbazine treatment improves survival in patients with metastatic and unresectable malignant pheochromocytomas/ paragangliomas. Horm Cancer 8(2):108-118

2. Hadoux J, Terroir M, Leboulleux S, Deschamps F, Al Ghuzlan A, Hescot S, Tselikas L, Borget I, Caramella C, Deandreis D, Goere D, De Baere T, Schlumberger M, Baudin E (2017) Interferon-alpha treatment for disease control in metastatic pheochromocytoma/ paraganglioma patients. Horm Cancer 8(5-6):330-337

3. Vezzosi D, Do Cao C, Hescot S, Bertherat J, Haissaguerre M, Bongard V, Drui D, De La Fouchardiere C, Illouz F, BorsonChazot F, Djobo B, Berdelou A, Tabarin A, Schlumberger M, Briet C, Caron P, Leboulleux S, Libe R, Baudin E, For Comete-Cancer N (2018) Time until partial response in metastatic adrenocortical carcinoma long-term survivors. Horm Cancer 9(1):62-69

4. Pinto MP, Muñoz Medel M, Carrillo D et al (2018) Chilean registry for neuroendocrine tumors: a latin American perspective. Horm Cancer. https://doi.org/10.1007/s12672-018-0354-5 